\title{
Une page autographe de Lamiel retrouvée dans les papiers d'Abraham Constantin
}

Hélène de Jacquelot et Jean-Jacques Labia

\section{(2) OpenEdition}

Journals

Édition électronique

URL : http://journals.openedition.org/rief/674

DOI : $10.4000 /$ rief.674

ISSN : 2240-7456

Éditeur

Seminario di filologia francese

Référence électronique

Hélène de Jacquelot et Jean-Jacques Labia, « Une page autographe de Lamie/ retrouvée dans les papiers d'Abraham Constantin », Revue italienne d'études françaises [En ligne], 4 | 2014, mis en ligne le 15 décembre 2014, consulté le 19 avril 2019. URL : http://journals.openedition.org/rief/674 ; DOI : $10.4000 /$ rief.674

Ce document a été généré automatiquement le 19 avril 2019

\section{c)}

Les contenus de la RIEF sont mis à disposition selon les termes de la Licence Creative Commons Attribution - Pas d'Utilisation Commerciale - Pas de Modification 4.0 International. 


\title{
Une page autographe de Lamiel retrouvée dans les papiers d'Abraham Constantin
}

\author{
Hélène de Jacquelot et Jean-Jacques Labia
}

1 Il n'est pas étonnant de trouver dans le fonds Abraham Constantin conservé à la Bibliothèque de Genève une page égarée de Lamiel. Au cours de l'hiver 1839-1840, outre son travail consulaire à Civitavecchia, Stendhal mène de front la reprise du manuscrit de Lamiel et la révision énergique des « Notices » de Constantin sur la peinture italienne. Pour ce faire il utilise plusieurs copistes, à Rome et à Civitavecchia, dont le copiste romain Falconi, appelé jusqu'ici «Corbeau » parce que Stendhal le mentionne par le dessin d'un oiseau qui, de fait, ressemble plus à un faucon ${ }^{1}$.

Lorsque, après son long congé parisien, Stendhal retourne en août 1839 à son poste de Civitavecchia, il retrouve à Rome son ami genevois, peintre sur porcelaine ${ }^{2}$, qui était là depuis fin janvier, chargé par le directeur de la Manufacture de Sèvres, Alexandre Brongniart, de copier sur porcelaine la fresque de la Délivrance de Saint Pierre des Chambres du Vatican ${ }^{3}$. Constantin comptait bien par ailleurs sur l'aide de son «ami Beyle » pour revoir les quelques Notices qu'il avait rédigées sur les tableaux italiens qu'il avait eu l'occasion de copier au cours de sa carrière et pour l'aider dans la correction des épreuves de ce petit ouvrage que le directeur du Cabinet Vieusseux, genevois comme lui, avait accepté de publier. Stendhal se passionne à la tâche et, sous son impulsion, à force de corrections et d'ajouts, ce petit ouvrage devient en quelques mois un volume de 358 pages qui sera publié à Florence en août 1840 : Idées italiennes sur quelques tableaux célèbres, un véritable guide de la peinture italienne et une nouvelle " promenade dans Rome ». Le manuscrit final se trouve au Cabinet Vieusseux, mais tous les avant-textes ont toujours été conservés par la famille d'Abraham Constantin qui, il y a quelques années, a légué à la Bibliothèque de Genève ces précieux documents qui illustrent cette écriture à quatre mains. C'est ainsi qu'une page de Lamiel s'est retrouvée par mégarde dans les papiers de Constantin. Une page à verser au complexe dossier génétique de ce roman inachevé. 
3 Cette page manuscrite de la main de StendhaI, doublement intitulée "Plan », est le témoin isolé de la genèse d'un passage de la scène du "miracle » des pétards, qui se déroule au cours de la mission racontée en début de roman. Cette scène ne se trouve pas dans la première version de Lamiel (L1). Elle fait l'objet de deux courts paragraphes dans le chapitre premier de la deuxième version, intitulée alors à son début L'Amiel, et qui date d'octobre-décembre 1839. Par contre dans la version suivante, intitulée Lamiel, datée en marge « $3 \mathrm{~J}[\mathrm{anvie}]^{\mathrm{r}} 40$ », un récit plus détaillé couvre une page entière du chapitre $\mathrm{II}^{4}$. Notre inédit se situe donc entre la deuxième et la troisième version du roman. Pour plus de clarté, sans entrer dans les détails complexes du dossier Lamiel, nous désignerons les trois versions successives, telles que proposées par la nouvelle édition de la Pléiade, respectivement par $L 1, L 2, L 3$.

4 Nous pouvons relever quatre éléments nouveaux introduits ici par Stendhal, absents de la deuxième version (L2) et qu'il maintient et étoffe dans la troisième (L3).

51 - Alors que les dévotes « s'étaient profondément évanouies » face à l'explosion alors que le missionnaire Le Cloud répétait « d'une voix criarde : "L’enfer, mes frères !" », l'extrême dévotion de Mme Hautemare se manifeste par le fait qu'elle « fut du nombre des plus évanouies » (L3, p. 972). Remarquons la reprise ironique, qui souligne la symétrie, dans le Plan du 3 janvier et ensuite dans L3, du même adverbe "profondément " pour les dévotes et pour M. Hautemare, " profondément occupé à rassembler les moindres lambeaux de l'enveloppe des pétards» (L3, p. 972).

62 - L'absence du bedeau Hautemare («M H » ici), chargé par le «terrible » (L3, p. 972) curé Du Saillard de la mission d'effacer les indices de la supercherie des pétards, est plus saillante dès lors que l'évanouissement de son épouse est lui-même devenu superlatif. Sa soumission et sa bêtise sont croquées : il comprend vite la consigne, à condition qu'on la lui explique plusieurs fois.

73 - L'abbé Du Saillard, « congrégationiste chargé de surveiller les curés du voisinage » (L3, p. 967), devient «un peu jaloux de l'immense succès obtenu par l'abbé Le Cloud» (L3, p. 973 ; orthographié Le Clou dans L2, et ici non nommé), le grand prédicateur venu d'ailleurs qui risque d'éclipser son aura locale à Carville. C'est cette jalousie qui le pousse à improviser un discours dans le cimetière où tout le monde est rassemblé : "il ne put résister à la tentation de monter sur le piédestal de la croix » (L3, p. 973). Ici, simplement sur une tombe, et « un peu jaloux».

84 « Le curé [...] se mit à signaler les impies ; [...] Ses yeux enflammés s'arrêtaient surtout sur trois personnes qui se trouvaient au cimetière, au milieu de bonnes femmes » ( $L 3 p$. 973). Désigner les impies qui se cacheraient parmi les femmes pieuses : L3 va développer cette nouvelle idée du «Plan » genevois en motivant l'agressivité de du Saillard par ses faiblesses en matière d'éloquence. Sur sa chaire improvisée, il n'est décidément pas à la hauteur de Le Cloud. Il convertit son impuissance en agressivité compensatoire. Notre page égarée lui attribue l'épithète homérique de « terrible ».

Un « Sommaire des chapitres », sans date, est commenté par une observation de l'auteur : «Usage des sommaires excellent pour les reprises du travail » (p. 1069-1070). Son contenu permet de le situer à coup sûr après $L 1$ et après notre page, puisqu'il mentionne à la fois « Le miracle, les pétards derrière l'autel » et le « discours de Du Saillard ». L'observation de l'auteur sur l'usage des sommaires s'applique parfaitement à la position de pivot qu'occupe l'inédit genevois, au moment précisément daté où Stendhal reprend le manuscrit de Lamiel, entre L2 et L3. La dictée de L3 comme version corrigée de L2 est 
entreprise en effet, comme l'indique L2, « le 3 Janvier $1840 »$ (note, p. 869), et c'est la date même qui figure ici en tête de notre page genevoise. La date du « 4 j[anvi]er [18]40", deuxième jour de la dictée de $L 3$ à partir de $L 2$, confirme cette interprétation en balisant sur le manuscrit de L3 (note, p. 978) le deuxième jour de cette campagne d'écriture.

\section{ANNEXES}

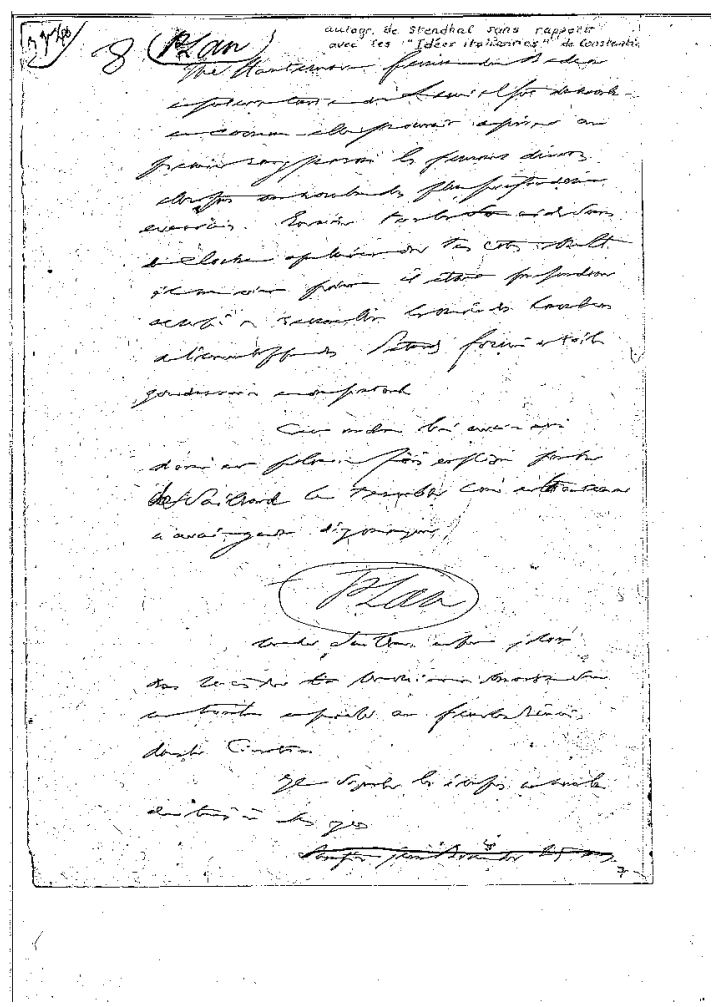

Bibliothèque de Genève, Ms. fr. 7222/3, f. 1, encre. Inédit.

$3 \mathrm{~J}^{\mathrm{er}} 40$

8 Plan

Mme Hautemare femme du bedeau et future tante de Lamiel fut du nombre et comme elle pouvait aspirer au premier rang parmi les femmes dévotes elle fut au nombre des plus profondément évanouies. En vain tous les son aides sonneurs de cloches appelaient de tous côtés $\mathrm{M} \mathrm{H}$ il ne vint point il était profondément occupé à rassembler les moindres lambeaux de [en surcharge sur du] l'enveloppe des Petars formée de toile 
goudronnée et de festonds

Cet ordre lui avait été

donné et plusieurs fois expliqué par $\mathrm{M}$

du Saillard le terrible curé et Hautemare

n'avait garde d'y manquer

Plan

M du Saillard un peu jaloux

ne resiste [illisible biffé] l'envie de monter sur

une tombe et parle aux femmes réunies

dans le cimetière.

Il signale les impies au nombre

de trois à ses gens

Sansfin jeune docteur de 25 ans

\section{NOTES}

1. Voir H. de Jacquelot, «Falconi ou la vraie identité du copiste prétendu "Corbeau" », http:// stendhal.msh-alpes.fr/manuscrits/index2.php?show=falconi (6 décembre 2014) et «Falconi, un des copistes romains de Lamiel et des Idées italiennes ", L'Année stendhalienne, de prochaine publication.

2. Sur les rapports de Stendhal avec Abraham Constantin voir l'édition des Idées italiennes sur quelques tableaux célèbres, S. Teroni et H. de Jacquelot éds., Paris, Beaux-Arts de Paris éditions, "D'art en questions ", 2013 et H. de Jacquelot, Stendhal et Constantin: une amitié féconde, dans Stendhal historien de l'art, Daniela Gallo éd., Rennes, PUR, 2012, p. 183-194.

3. Lettre d'Abraham Constantin à Alexandre Brongniart du 2 février 1839 : « [...] enfin arrivé à Rome le 28 janvier » (Inédit. Manufacture de Sèvres, T13 L6 d17).

4. Stendhal, Lamiel dans Euvres romanesques complètes, t. 3, Y. Ansel, Ph. Berthier, S. Linkès (éds.), Gallimard, «Bibliothèque de la Pléiade», p. 869 et 972-973. Toutes les références renvoient à cette édition. Notre page est bien entendu postérieure au brouillon du 2 octobre (Ibidem, p. 956).

\section{RÉSUMÉS}

Une page autographe de Lamiel retrouvée dans les papiers d'Abraham Constantin enrichit le dossier génétique complexe de ce roman inachevé.

\section{INDEX}

Mots-clés : Lamiel, Stendhal, manuscrit, inédit 